\title{
Review of: "Predictive model of pheochromocytoma based on the imaging features of the adrenal tumours"
}

Partha P Chakraborty

Potential competing interests: The author(s) declared that no potential competing interests exist.

Comment 1:(Figure 1)

$8+25+305+576=914$

What was the final diagnosis in the remaining 54 patients?

Comment 2: (Figure 1 legend)

These numbers are different from the figures, mentioned in the boxes (Figure 1). Please explain.

Comment 3:(Table 1) How did the authors define obesity?

Comment 4:(Table 2) Does 'high lipid content' mean those with NCCT HU of less than 10?

Comment 5:(Discussion)

What would be the expected non-contrast HU in areas with fatty degeneration? does it explain the discrepancy between \% of PHEO patients having HU of more than 10 and \% of PHEO patients with loss of signal in out of phase MRI image?

Comment 6: Discuss in brief the ideal protocol of measuring HU (like size of ROI in relation to the size of the mass, placing the ROI over areas without necrosis/haemorrhage, preferable an average of 3 values obtained from different homogenous areas of the mass etc) in paragraph mentioning the limitations of the study 